\title{
A Valve in Valve: Mitral Valve Replacement with Tricuspid Annuloplasty in a Case of Mitral Annular Calcification
}

Mohammed Idhrees A*, Vargheese T Panicker, Bineesh K Radhakrishnan, Vivek Pillai and Jayakumar Karunakaran

Department of Cardiovascular and Thoracic Surgery, Sree Chitra Thirunal Institute for Medical Sciences and Technology, Thiruvananthapuram, Kerala, India

\section{Case Report}

A 79 year old female presented with history of dyspnoea on exertion (New York Hear Association class III) and palpitation at rest for last 3 years and has developed paroxysmal nocturnal dyspnoea during the last 1 year. On examination she had irregular pulse. She had a hyper dynamic precordium with the apex displaced to the left $6^{\text {th }}$ intercoastal space $1 \mathrm{~cm}$ lateral to the midclavicular line. A pan systolic murmur was heard at the apex. Chest X-ray showed annular calcification of the mitral valve. Echocardiogram revealed a dilated left ventricle (LVEDD $69 \mathrm{~mm}$ ). There was bi-leaflet prolapse causing severe mitral regurgitation. Tricuspid annulus was dilated $(43 \mathrm{~mm})$ causing moderate regurgitation (Figure 1). There was a mild pulmonary artery hypertension. She was planned for surgery and under moderate hypothermic cardiac arrest; left atrium was approached through the Sondergaard's groove. We can appreciate calcification of the mitral annulus extending on the posterior annulus across both commisures and partly into the anterior annulus. It was also extending into the ventricular mass. Mitral valve was replaced with \#25 bioprosthetic valve with complete preservation of both the leaflets. Plegetted sutures taken from atrium to ventricle through the leaflets in a way to plicate them were used to fix the prosthetic valve. Tricuspid annuloplasty ring was placed. She had a normal postoperative recovery. Her echocardiogram at discharge and 6 month follow up showed no paravalvular leak and good function of the prosthetic valve (Figure 2).

Mitral annular calcification (MAC) continues to be a challenge in valvular heart surgery and associated with high rate of complication including atrioventricular groove rupture, myocardial infraction, haemorrhage, ventricular perforation, paravalvular leak [1]. Carpentier [2] classified MAC based on its circumferential extension around the mitral annulus and the radial extension into the leaflets and ventricle. There are various techniques described to treat MAC with mitral valve pathology. These include procedure with mitral annular reconstruction after decalcification or without decalcification and the mitral valve

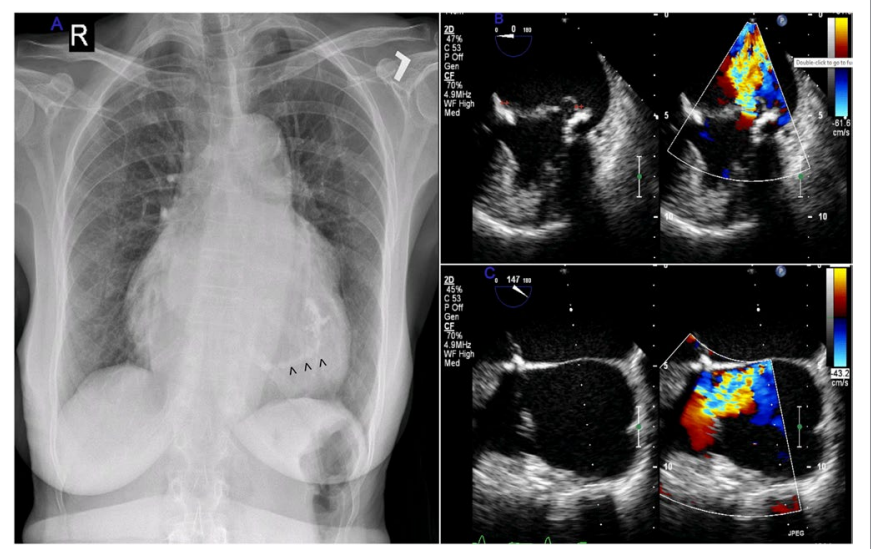

Figure 1: (A) Chest $\mathrm{X}$-ray showing mitral annular calcification (arrow heads). (B) Tranesophageal Echocardiography showing severs mitral regurgitation. $(++)$ mark indicating mitral annular calcification. (C) Tranesophageal echocardiography showing moderate tricuspid regurgitation.

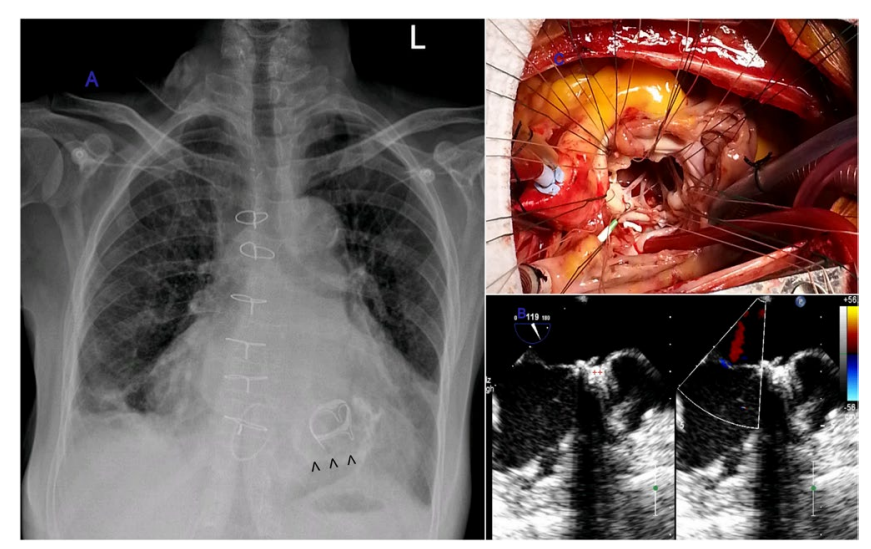

Figure 2: (A) Postoperative Chest X-ray showing mitral annular calcification with bioprosthetic mitral valve. Note the space between the mitral annular calcification and valve which is actually the plicated native mitral leaflets. Also the tricuspid valve annuloplasty ring can be seen. (B) Intraoperative picture showing the valvular suture taken from atrium to ventricle placating the leaflets as well. (C) Postoperative Tranesophageal Echocardiography showing the bioprosthetic valve in mitral position, also the mitral annular calcification can be visualised (++).

are either repaired or replaced [3]. Procedures with delcalification and reconstruction have higher rate of atrioventricular dissociation unless done in expertised hand. Procedures without decalcification have higher incidence of paravalvular leak and haemorrhage. Valve in valve without decalcification still continues to be a safe procedure with minimal morbidity and low paravalvular leak if executed meticulously.

\section{References}

1. Yukikatsu Okada (2013) Surgical Management of mitral annular calcification. Gen Thorac Cardiovasc Surg 61: 619-625.

2. Carpentier A, Pellerin M, Fuzellier JF, Relland J (1996) Extensive calcification of mitral valve annuls: Pathology and surgical management. J Thorac Cardiovas Surg 111: 718-730.

3. Vidyadhar S, Andrew E Newcomb, Philip J Davis, Chua Yeow Leng (2014) Surgical Techinques for management of the "hostalie mitral annulus". Heart, Lung and Circulation 23: 217-223.

*Corresponding author: Mohammed Idhrees A, Senior Resident, Department of Cardiovascular and Thoracic Surgery, Sree Chitra Thirunal Institute for Medical sciences and Technology, Thiruvananthapuram-695011, Kerala, India, Tel: +91 9962268787; Fax: 91-471-2446433; E-mail: a.m.idhrees@gmail.com

Received November 16, 2015; Accepted December 10, 2015; Published December 15, 2015

Citation: Idhrees AM, Panicker VT, Radhakrishnan BK, Pillai V, Karunakaran J (2015) A Valve in Valve: Mitral Valve Replacement with Tricuspid Annuloplasty in a Case of Mitral Annular Calcification. Cardiovasc Pharm Open Access 4: 166. doi:10.4172/2329-6607.1000166

Copyright: (c) 2015 Idhrees AM, et al. This is an open-access article distributed under the terms of the Creative Commons Attribution License, which permits unrestricted use, distribution, and reproduction in any medium, provided the original author and source are credited. 